\title{
The Exterior Plateau Problem in Higher Codimension
}

\author{
Luquésio P. Jorge * $\quad$ Friedrich Tomi**
}

\begin{abstract}
We prove existence theorems for two-dimensional non-compact complete minimal surfaces in $\mathbb{R}^{n}$ of annular type which span a given contour and have a finite total curvature end and prescribed asymptotical behaviour. For arbitrary rectifiable Jordan curves we show the existence of such surfaces with a flat end, i. e. within bounded distance from a 2-plane. For more restricted classes of curves we prove the existence of minimal surfaces with higher multiplicity flat ends as well as of surfaces with polynomial type non-flat ends.
\end{abstract}

\section{Introduction}

Let $\Gamma$ be a rectifiable Jordan curve in $\mathbb{R}^{n}$. The exterior Plateau problem for $\Gamma$ asks for a complete non-compact minimal surface spanning $\Gamma$. While geometric measure theory may easily provide the existence of solutions of unspecified topological type to this problem, we in this paper are interested in finding surfaces of annular type which moreover have a specified asymptotical behaviour at infinity. Whereas embedded finite total curvature ends of minimal surfaces in $\mathbb{R}^{3}$ must be asymptotic to a plane or to a half catenoid, there is a large variety of different such ends in $\mathbb{R}^{n}$ as soon as $n \geq 4$ : algebraic complex curves in a complex subspace of $\mathbb{R}^{n}$ yield plenty of examples. This makes the higher codimensional Plateau problem even more interesting than the corresponding problem in $\mathbb{R}^{3}$ which was investigated by $\mathrm{R}$. Ye and one of the present authors $[\mathrm{T}-\mathrm{Y}]$. Though in higher codimension one may construct many candidates of solutions of different asymptotic behaviour, it seems to be quite difficult to actually verify their behaviour at infinity, apart from the simplest case of a flat end. This difficulty is due to the lack of the usual comparison principle for codimension one mimimal surfaces. Instead we must rely on a more general barrier principle for minimal surfaces of arbitrary codimension which we established in a previous paper [J-T]. So far, our supply of useful and non-trivial barriers is however rather limited, due to their cumbersome construction.

The plan of the paper is as follows. In section 2 we first prove an existence result for compact minimal annuli which is based on Morgan's characterization of area minimizing sums of two

\footnotetext{
*Departamento de Matemática da Universidade Federal do Ceará, Campus do Pici, 60455-760 Fortaleza-Ce Brazil. Work done during a visit to the Mathematisches Institut at the Universität Heidelberg, supported by CAPES and UFC.

** Mathematisches Institut, Universität Heidelberg, Im Neuenheimer Feld 288, D-69120 Heidelberg, Federal Republic of Germany
} 
2-planes in $\mathbb{R}^{n}[\mathrm{M}]$. We then apply this to construct families of expanding compact minimizing annuli spanning a fixed "inner" Jordan curve $\Gamma$ and an "outer" Jordan curve from a family $\Gamma_{R}, R \geq R_{0}$, which is obtained as the intersection of a model surface $M_{0}$ with spheres of radius $R$. Here we assume that $M_{0}$ is a complete simply connected, area-minimizing, finite total curvature minimal surface with an embedded end and that the asymptotical tangent plane of $M_{0}$ is not orthogonal to the affine subspace of minimal dimension containing $\Gamma$. Subsequently, we show that there are sequences $R_{k} \rightarrow+\infty(k \rightarrow \infty)$ such that the minimizing annuli spanning $\Gamma \cup \Gamma_{R_{k}}$ converge to a limit surface when $k \rightarrow \infty$. In section 3 we investigate the asymptotical behaviour of the limit surfaces and show that they are complete and have a finite total curvature end. In the case that $M_{0}$ is a plane we prove that the limit surfaces have a flat end of geometric index 1, the asymptotic tangent plane having the direction of $M_{0}$. In section 4 , we apply our barrier principle $[\mathrm{J}-\mathrm{T}]$ and the concept of incompressible surfaces to establish the existence of complete finite total curvature surfaces with a flat end of geometric index $m>1$ spanning the Jordan curve $\Gamma$ provided that $\Gamma$ is contained in the non-simply connected domain bounded by one of our catenoidal barriers and has the winding number $m$. Finally, in section 5 we construct a barrier around the end of the model surface $M_{0}=\left\{(z, w) \in \mathbb{C} \times \mathbb{C} \mid w=z^{m}\right\}, m \in \mathbb{N}$, which is contained in a distance 1 neighborhood of $M_{0}$. This immediately leads to an existence theorem for solutions to Plateau's problem asymptotic to this $M_{0}$.

Some of the arguments in the sections 2 and 3 of this paper are identical with those in the codimension 1 case treated in [T-Y], some arguments are essentially different. For instance, the curvature estimates used in $[\mathrm{T}-\mathrm{Y}]$ are not available in codimension $>1$. We present the complete proofs in order to make the paper better readable.

\section{The Expanding Minimal Annuli}

The basis of our construction is Morgan's characterization of the singularity structure of twodimensional area minimize surfaces in $\mathbb{R}^{n}[\mathrm{M}]$. We use this characterization in the following Lemma to verify Douglas' condition for the existence of least area annuli spanning a given pair of Jordan curves in $\mathbb{R}^{n}[\mathrm{D}]$.

Let us introduce some notation:

$$
\begin{aligned}
D_{R}: & =\left\{u=\left(u_{1}, u_{2}\right) \in \mathbb{R}^{2}|| u \mid \leq R\right\}, R>0, \\
A_{R}: & =D_{R} \backslash \operatorname{int}\left(D_{1}\right) \quad R>1, \\
B_{\rho}(p): & =\left\{x \in \mathbb{R}^{n}|| x-p \mid \leq \rho\right\}, p \in \mathbb{R}^{n}, \rho>0 .
\end{aligned}
$$

Lemma 2.1 Let $M_{1}, M_{2} \subset \mathbb{R}^{n}$ be classical disc type least area surfaces spanning the rectifiable Jordan curves $\Gamma_{1}$ and $\Gamma_{2}$, respectively, i.e. $M_{1}$ and $M_{2}$ are the images of continuous maps $x_{k}: D_{1} \rightarrow \mathbb{R}^{n}, k=1,2$, where $x_{k} \mid \partial D_{1}$ parameterizes $\Gamma_{k}, x_{k}$ is a harmonic conformal branched immersion in the interior of $D_{1}$, and $x_{k}$ minimizes area among all maps $y \in C^{0}\left(D_{1}, \mathbb{R}^{n}\right) \cap$ 
$C^{0,1}\left(\stackrel{\circ}{D}_{1}, \mathbb{R}^{n}\right)$ such that $y \mid \partial D_{1}$ parameterizes $\Gamma_{k}$ weakly monotonically. We assume furthermore that there are points $w_{i} \in \stackrel{\circ}{D}_{1}, k=1,2$, such that $w_{k}$ is regular for $x_{k}, x_{1}\left(w_{1}\right)=x_{2}\left(w_{2}\right)$, and the tangent spaces $T_{k}$ of $x_{k}$ at $w_{k}$ are not mutually orthogonal. Then there is an orientation $\mathcal{O}$ of $\Gamma_{1} \cup \Gamma_{2}$ and a number $\delta>0$ such that

$$
a\left(\Gamma_{1} \cup \Gamma_{2}, \mathcal{O}\right) \leq \operatorname{area}\left(M_{1}\right)+\operatorname{area}\left(M_{2}\right)-\delta,
$$

where $a\left(\Gamma_{1} \cup \Gamma_{2}, \mathcal{O}\right)$ denotes the infinium of the area of all mappings $y \in C^{0}\left(A_{2}, \mathbb{R}^{n}\right) \cap$ $C^{0,1}\left(\stackrel{\circ}{A_{2}}, \mathbb{R}^{n}\right)$ such that $y \mid \partial A_{2}$ parameterizes $\Gamma_{1} \cup \Gamma_{2}$ coherent with the orientation $\mathcal{O}$. Hence, it follows from Douglas' theorem $[D]$ that $\Gamma_{1} \cup \Gamma_{2}$ with the chosen orientation $\mathcal{O}$ spans a classical least area annulus, i.e. there are a number $r>1$ and a harmonic, conformal, branched immersion $x$ of $\stackrel{\circ}{A_{r}}$ into $\mathbb{R}^{n}$ which is continuous on $A_{r}$ and such that $x \mid \partial A_{r}$ parameterizes $\Gamma_{1} \cup \Gamma_{2}$ coherent with $\mathcal{O}$ and area $(x)=a\left(\Gamma_{1} \cup \Gamma_{2}, \mathcal{O}\right)$.

Proof: We may assume that $x_{1}\left(w_{1}\right)=x_{2}\left(w_{2}\right)=0$, the origin of our coordinate system.

Let us denote by $\Delta_{k}(\rho) \subset T_{k}$ the discs of radius $\rho$ and center 0 . Since by assumption $T_{1}$ and $T_{2}$ are not orthogonal, it follows from Corollary 6 of [M] that the discs $\Delta_{1}(1)$ and $\Delta_{2}(1)$ can be oriented in such a way that $\Delta_{1}(1)+\Delta_{2}(1)$ is not area minimizing in the class of oriented surfaces. We choose such an orientation $\mathcal{O}$ of $\Delta_{1}(1)$ and $\Delta_{2}(1)$ and, if necessary, we reorient the surfaces $x_{1}$ and $x_{2}$ in such a way that the induced orientation of the tangent spaces $T_{1}$ and $T_{2}$ coincides with $\mathcal{O}$. Beyond the statement of Morgan's Corollary 6 we need the following more precise information from the proof of Theorem 2 in the same paper: there is a smooth annulus type surface $C$ with $\partial C=\partial \Delta_{1}(1)+\partial \Delta_{2}(1)$ and area $(C)<2 \pi$. Since $x_{k}$ is an embedding near $w_{k}$ for $k=1,2$ there are neighborhoods $U_{k}$ of $w_{k}$ and $\rho_{o}>0$ such that $x_{k}\left(U_{k}\right)$ has a one-to-one orthogonal projection onto $\Delta_{k}\left(\rho_{o}\right) \subset T_{k}$. Let us denote by $M_{k}(\rho)$ the piece of $x_{k}\left(U_{k}\right)$ which projects onto $\Delta_{k}(\rho) ; k=1,2 ; 0<\rho \leq \rho_{o}$. By the smoothness of $x_{k}$ the vertical height of $M_{k}(\rho)$ over $\Delta_{k}(\rho)$ is estimated by const. $\rho^{2}$ and we may therefore construct cylindrical annuli $C_{k}(\rho)$ with $\partial C_{k}(\rho)=\partial M_{k}(\rho)-\partial \Delta_{k}(\rho)$ and area $\left(C_{k}(\rho)\right) \leq$ const. $\rho^{3}$. It follows that $S_{\rho}:=C_{1}(\rho)+\rho C+C_{2}(\rho)$ is an annulus with boundary $\partial S_{\rho}=\partial M_{1}(\rho)+\partial M_{2}(\rho)$ and area $\left(S_{\rho}\right) \leq \rho^{2}$ area $\left(C_{1}(1)\right)+$ const. $\rho^{3}$. Since area $\left(C_{1}(1)\right)<2 \pi$ we may find $\rho>0$ and $\delta>0$ such that area $\left(S_{\rho}\right) \leq 2 \pi \rho^{2}-\delta$ and hence

$$
\operatorname{area}\left(S_{\rho}\right) \leq \operatorname{area}\left(M_{1}(\rho)\right)+\operatorname{area}\left(M_{2}(\rho)\right)-\delta .
$$

We may now delete the $\operatorname{discs} M_{k}(\rho)$ from $M_{k}$ and join the resulting annuli $M_{k}-M_{k}(\rho)$ by $S_{\rho}$ to obtain an annulus $M_{\rho}$ with $\partial M_{\rho}=\Gamma_{1} \cup \Gamma_{2}$ and, as follows immediately from (2.2),

$$
\operatorname{area}\left(M_{\rho}\right) \leq \operatorname{area}\left(M_{1}\right)+\operatorname{area}\left(M_{2}\right)-\delta \text {. }
$$

Remark 2.2 As the above proof shows, the number $\delta$ in the Douglas condition(2.1) depends only on the restriction of the surfaces $x_{k}$ onto some neighborhood of $w_{k}(k=1,2)$. 
We shall now apply Lemma 2.1 in the situation when $M_{1}$ is a fixed least area disc $M$ spanning the given Jordan curve $\Gamma$ and $M_{2}$ is the intersection of a model surface $M_{0}$ with a ball of radius $R$, denoted by $M_{0}^{R}$. We assume the following about $M_{0}: M_{0}$ is a complete, simply connected, finite total curvature minimal surface with an embedded end and all compact subdiscs of $M_{0}$ are area-minimizing among disc type surfaces. Complex algebraic curves in some complex subspace of $\mathbb{R}^{n}$ yield plenty of examples of such model surfaces.

It follows from the above properties of $M_{0}$ that $M_{0}$ possesses a limit tangent space at infinity which we denote by $T$.

Let then $\Gamma \subset \mathbb{R}^{n}, n \geq 4$, be a rectifiable Jordan curve contained in the unit ball of $\mathbb{R}^{n}$, and let $V \subset \mathbb{R}^{n}$ be the affine subspace of $\mathbb{R}^{n}$ of minimal dimension containing $\Gamma$. Let $M$ denote a classical least area disc spanning $\Gamma$. By the convex hull property of minimal surfaces, $M$ is contained in $V$ and by the minimality of $\operatorname{dim} V$ the tangent spaces of $M$ generate the tangent space of $V$. Hence, if the asymptotic tangent space of $T$ of $M_{0}$ is not orthogonal to $V$, there is an open set of points on $M$ whose tangent spaces are not orthogonal to $T$. We may therefore choose a regular point $p$ on $M$ and a regular point $q$ on $M_{0}$ such that their tangent spaces are not orthogonal. After a translation of $M_{0}$ we may assume that $p=q$. We finally observe that $\Gamma_{R}:=\partial M_{0}^{R}$ is a smooth Jordan curve for $R \geq R_{0}>1$ since the end of $M_{0}$ is embedded by assumption. From Lemma 2.1 and Remark 2.2 we therefore obtain

Lemma 2.3 Let $\Gamma \subset B_{1}(0)$ be a rectifiable Jordan curve, and $M_{0}$ as described above, and assume that the subspace of minimal dimension containing $\Gamma$ is not orthogonal to $T$, the asymptotic tangent space of $M_{0}$. Then, after translating and possibly reorienting $M_{0}$ there exist, for any $R>R_{0}$, classical least area annuli $M_{R}$ spanning $\Gamma \cup \Gamma_{R}$. The annuli $M_{R}$ are given by harmonic, conformal, branched immersions $x_{R}: \stackrel{\circ}{A}_{r(R)} \rightarrow \mathbb{R}^{n}$, where $x_{R}$ is continuous on $A_{r(R)}$ and $x_{R} \mid \partial A_{r(R)}$ maps $\partial A_{r(R)}$ topologically onto $\Gamma \cup \Gamma_{R}$, coherent with the chosen orientation of $\Gamma \cup \Gamma_{R}$. Moreover, there is a number $\delta>0$ such that the inequalities

$$
\operatorname{area}\left(M_{R}\right) \leq \operatorname{area}(M)+\operatorname{area}\left(M_{o}^{R}\right)-\delta
$$

hold for all $R>R_{0}$, where $M$ is a least area disc spanning $\Gamma$.

By a well know cut-and-paste argument [T-T] one infers from the uniform Douglas-condition (2.3) a uniform "condition of cohesion".

Corollary 2.4 There is a number $\varepsilon>0$ such that the length of the images under any $x_{R}$ of all homotopically non-trivial loops in $A_{r(R)}$ is bounded below by $\varepsilon$. One may take any $\varepsilon<\sqrt{\delta}$.

Now we are going to establish uniform (with respect to $R$ ) local area estimates for the surfaces $M_{R}$. These follow easily from the monotonicity formula for minimal surfaces with boundary [E-W-W]: let $M$ be any minimal surface in $\mathbb{R}^{n}$ with $C^{1}$ boundary $\partial M$ and for $p \in \mathbb{R}^{n}$ and $\rho>0$ let us define 


$$
M_{\rho}:=M \cap B_{\rho}(p), \quad(\partial M)_{\rho}:=\partial M \cap B_{\rho}(p), \quad d(x):=|x-p| .
$$

Then the following formula holds:

$$
\frac{\partial}{\partial \rho}\left(\frac{\operatorname{area}\left(M_{\rho}\right)}{\rho^{2}}\right) \geq-\frac{1}{\rho^{3}} \int_{(\partial M)_{\rho}} d\langle\nabla d, \nu\rangle d s,
$$

where $\nu$ denotes the unit exterior surface normal along $\partial M$. If $(\partial M)_{\rho}$ is contained in $B_{r}(p)$ we obtain the estimate

$$
\frac{\partial}{\partial \rho}\left(\frac{\operatorname{area}\left(M_{\rho}\right)}{\rho^{2}}\right) \geq-\frac{r}{\rho^{3}} \operatorname{length}(\partial M)_{\rho} .
$$

Let us apply this formula to our annuli with $p=0$ and $1<\rho<R$ such that $(\partial M)_{\rho}=\Gamma$ and consequently $r \leq 1$. By integration we obtain

$$
R^{-2} \text { area }\left(M_{R}\right)-\rho^{-2} \operatorname{area}\left(\left(M_{R}\right)_{\rho}\right) \geq\left(\frac{1}{2} R^{-2}-\frac{1}{2} \rho^{-2}\right) \operatorname{length}(\Gamma)
$$

It is well known, that $M_{0}$ has quadratic area growth, i.e. there exist positive numbers $a$ and $m$ such that,

$$
\operatorname{area}\left(M_{0}^{R}\right) \leq a+m \pi R^{2} .
$$

Combining this with (2.3) and (2.4) we obtain

Lemma 2.5 The annuli $M_{R}$ fulfill the estimate

$$
\operatorname{area}\left(\left(M_{R}\right)_{\rho}\right) \leq \gamma+m \pi \rho^{2}, \quad 1 \leq \rho \leq R
$$

with $\gamma:=\operatorname{area}(M)+1 / 2$ length $(\Gamma)+a$.

Completely analogous to the proceeding in $[\mathrm{T}-\mathrm{Y}]$ we may now control the conformal parameterization $x_{R}: A_{r(R)} \rightarrow \mathbb{R}^{n}$ of $M_{R}$. Because of the conformality the area of $x_{R}$ over any domain $G$ coincides with the Dirichlet energy. 


$$
E\left(x_{R}, G\right):=\frac{1}{2} \iint_{G}\left(\left|\frac{\partial}{\partial u_{1}}\right|^{2}+\left|\frac{\partial}{\partial u_{2}}\right|^{2}\right) d u_{1} d u_{2}
$$

We introduce the following subdomains of the parameter domain $A_{r(R)}$ :

$$
\begin{aligned}
& \Omega\left(x_{R}, \rho\right):=\left(x_{R}\right)^{-1}\left(B_{\rho}\right) \\
& A\left(x_{R}, \rho\right):=\text { the component of } \Omega\left(x_{R}, \rho\right) \text { containing } \partial D_{1}=S^{1}
\end{aligned}
$$

\section{Lemma 2.6}

(i) $\operatorname{dist}\left(\partial A\left(x_{R}, \rho\right) \backslash S^{1}, \partial A\left(x_{R}, 2 \rho\right) \backslash S^{1}\right) \geq \exp (-4 \pi(\gamma+m \pi)), 1<\rho<R / 2$

(ii) $\operatorname{dist}\left(O, \partial A\left(x_{R}, \rho\right)\right) \geq c \ln \rho$ for some positive constant $c, 1<\rho<R$

(iii) $r(R) \geq c \ln r$

Proof: Let us choose a point $z \in \partial A\left(x_{R}, \rho\right) \backslash S^{1}$ such that

$$
\operatorname{dist}\left(z, \partial A\left(x_{R}, 2 \rho\right) \backslash S^{1}\right)=r_{o}:=\operatorname{dist}\left(\partial A\left(x_{R}, \rho\right) \backslash S^{1}, \partial A\left(x_{R}, 2 \rho\right) \backslash S^{1}\right) .
$$

We need only consider the case when $r_{o}<1$. Let us define $D_{r}(z):=\left\{u \in \mathbb{R}^{2}|| u-z \mid \leq r\right\}$ and $\partial^{*} D_{r}(z):=\partial D_{r}(z) \cap A\left(x_{R}, 2 \rho\right)$. According to the Lemma of Courant-Lebesgue [C, p. 101] there exists $r_{1} \in\left(r_{o}, \sqrt{r_{o}}\right)$ such that

$$
\begin{aligned}
\left(\int_{\partial^{*} D_{r_{1}(z)}}\left|\frac{\partial x_{R}}{\partial \theta}\right| d \theta\right)^{2} & \leq \frac{4 \pi}{-\ln r_{o}} E\left(x_{R}, A\left(x_{R}, 2 \rho\right)\right) \\
& \leq-\frac{4 \pi}{\ln r_{o}} \operatorname{area}\left(\left(M_{R}\right)_{2 \rho}\right)
\end{aligned}
$$

Since $r_{1} \geq r_{o}$ and by the definition of $A\left(x_{R}, 2 \rho\right)$ there must be an arc in $\partial^{*} D_{r_{1}}(z)$ connecting $\partial A\left(x_{R}, 2 \rho\right)$ with $\Omega\left(x_{R}, \rho\right)$. Hence the left hand side of (2.5) has the lower bound $\rho^{2}$ and Lemma 2.5 immediately gives $(i)$. Assertion $(i i)$ is an easy consequence of $(i)$ (cf. Lemma 2.4 of [T-Y]) and $(i i i)$ follows trivially from $(i i)$.

Lemma 2.5 and Lemma 2.6 yield directly the

\section{Corollary 2.7}

(i) $\sup \left|x_{R}\right| \leq e^{r / c}$

(ii) $\stackrel{A_{r}}{E}\left(x_{R}, A_{r}\right) \leq \gamma+m \pi e^{2 r / c}, \quad 1<r<r(R)$. 
We may now prove the existence of limit surfaces to our family of least area annuli. We define $A:=\left\{u \in \mathbb{R}^{2}|| u \mid \geq 1\right\}$.

Proposition 2.8 For every sequence $R_{k} \rightarrow+\infty(k \rightarrow \infty)$ there is a subsequence (again denoted by $\left.R_{k}\right)$ and a map $x \in C^{0}\left(A, \mathbb{R}^{n}\right) \cap C^{\infty}\left(\stackrel{\circ}{A}, \mathbb{R}^{n}\right)$ with the following properties:
(i) $x \mid \stackrel{\circ}{A}$ is a harmonic, conformal branched immersion,
(ii) $x \mid \partial A$ maps $\partial A$ topologically onto $\Gamma$,
(iii) $\quad x_{R_{k}} \rightarrow x(k \rightarrow \infty)$ in $C^{\infty}(\stackrel{\circ}{A})$, in $C^{0}\left(A_{r}\right)$, and weakly in $W^{1,2}\left(A_{r}\right)$ for every $r>1$.

Proof: The existence of a subsequence of $\left(R_{k}\right)$ and a limit map $x: \stackrel{\circ}{A} \rightarrow \mathbb{R}^{n}$ such that $x_{R_{k}} \rightarrow x$ in $C^{\infty}(\stackrel{o}{A})$ and weakly in $W^{1,2}\left(A_{r}\right)$ for every $r$ follows immediately from Corollary 2.7. The map $x$ must be harmonic and conformal and, unless it is constant, a branched immersion as a $C^{\infty}$-limit of maps with these properties. From the local energy bound (Corollary 2.7) and the condition of cohesion (Corollary 2.4) we obtain by a well-know argument (cf. T-T) the uniform continuity of the sequence $\left(x_{R_{k}} \mid S^{1}\right)$. It follows that $x$ extends as a continuous map on $A$ and that $x$ maps $\partial A$ weakly monotonically onto $\Gamma$, in particular, $x$ is not constant. Since $x$ is conformal and harmonic, $x \mid \partial A$ must actually be a topological map.

\section{The asymptotical behavior of the limit surfaces}

In this section we investigate further the properties of a minimal surface $x: A \rightarrow \mathbb{R}^{n}$ which is obtained by the limiting process of Proposition 2.8. In particular, we would like to show that $x$ has a properly immersed finite total curvature end. Finally, we prove our first main result, Theorem 3.5 about solutions with a flat end as announced in the introduction.

Lemma $3.1 E(x, \Omega(x, \rho)) \leq \gamma+m \pi \rho^{2}, \quad \rho>1$.

Proof: Let $C$ be any compact subset of $\stackrel{\Omega}{\Omega}(x, \rho)$. Because of the convergence $x_{R_{k}} \rightarrow x, k \rightarrow \infty$, we have $C \subset \Omega\left(x_{R_{k}}, \rho\right)$ for sufficiently large $k$ and Lemma 2.5 yields

$$
\begin{aligned}
E(x, C) & \leq \liminf _{k \rightarrow \infty} E\left(x_{R_{k}}, C\right) \\
& \leq \gamma+m \pi \rho^{2} .
\end{aligned}
$$

The assertion of the lemma follows.

Lemma 3.2 Each component of $\Omega(x, \rho)$ is a bounded subset of $A$ for any $\rho>1$.

Proof: (cf. $[\mathrm{T}-\mathrm{Y}]$ ) Let us first show that $\Omega(x, \rho) \neq A$ for any $\rho$, i.e. that $x$ is not bounded. We consider the map $x^{*}: D_{1}^{*} \rightarrow \mathbb{R}^{n}, D_{1}^{*}:=D_{1} \backslash\{0\}, x^{*}(z):=x(1 / z)$. If $x$ were bounded, so were $x^{*}$ and hence $x^{*}$ had a removable singularity at the origin. It follows that the length of $x^{*}\left(\partial D_{r}\right)=x\left(\partial D_{1 / r}\right)$ converges to 0 when $r \rightarrow 0$, whence the length $\left(x_{R_{k}}\left(\partial D_{\rho}\right)\right)$ becomes arbitrarily small for large $k$ and $\rho$, contradicting Corollary 2.4. 
Let us now assume that $\Omega_{o}$ is an unbounded component of $\Omega(x, \rho)$ for some $\rho$. Let us choose a regular value $\rho^{\prime}$ of $d \circ x=|x|, \rho^{\prime}>\rho$, and let $\Omega^{\prime}$ denote the component of $\Omega\left(x, \rho^{\prime}\right)$ containing $\Omega_{o}$. Then $\Omega^{\prime}$ is also unbounded and hence $\partial \Omega^{\prime} \backslash S^{1}$ consists of finitely many properly embedded unbounded analytic curves. Let us again consider $x^{*}$ instead of $x$ and let $\Omega^{*}$ be the image of $\Omega^{\prime}$ under the map $z \mapsto 1 / z$. From Lemma 3.1 and the invariance of Dirichlet's integral under conformal maps it follows that $E\left(x^{*}, \Omega^{*}\right)$ is finite and we may hence apply the lemma of Courant-Lebesgue [C ] to find a radius $r, 0<r<1$, such that length $\left(x^{*}\left(\Omega^{*} \cap \partial D_{r}\right)\right)<\rho^{\prime}-\rho$. Since any component of $\Omega^{*} \cap \partial D_{r}$ must meet $\partial \Omega^{*}$ we conclude that $\left|x^{*}\right|>\rho$ on $\Omega^{*} \cap \partial D_{r}$. Hence $\Omega^{\prime} \cap A_{1 / r}$ is a bounded set such that $|x|>\rho$ on $\partial\left(\Omega^{\prime} \cap A_{1 / r}\right) \backslash S^{1}$. We may choose $r$ so small that $\Omega^{\prime} \cap A_{1 / r}$ contains some point of $\Omega_{o}$. It follows then that $\Omega_{o} \subset \Omega^{\prime} \cap A_{1 / r}$ and hence $\Omega_{o}$ is bounded.

Proposition 3.3 The map $x$ is proper, i.e. $|x(u)| \rightarrow+\infty$ for $|u| \rightarrow+\infty$.

Proof: Let $\rho>1$ be arbitrarily chosen and let $\Omega_{1}, \Omega_{2}, \ldots$ denote those components of $\Omega(x, 2 \rho)$ which are different from $A(x, 2 \rho)$ and contain some point $w_{k} \in \Omega_{k}$ with $\left|x\left(w_{k}\right)\right|<\rho$. Then $x\left(\Omega_{k}\right)$ is a disc type minimal surface which has no boundary in the interior of the ball $B_{\rho}\left(x\left(w_{k}\right)\right)$. It follows from the monotonicity formula (cf. section 2 ) that $E\left(x, \Omega_{k}\right) \geq \pi \rho^{2}$. In view of Lemma 3.1 we conclude that there can exist only finitely many such $\Omega_{k}$, say $\Omega_{1}, \ldots, \Omega_{l}$. On the complement of $A(x, 2 \rho) \cup \Omega_{1} \cup \ldots \cup \Omega_{l}$ we have the inequality $|x| \geq \rho$ and hence $\Omega(x, \rho) \subset A(x, 2 \rho) \cup \Omega_{1} \cup \ldots \cup \Omega_{l}$. The latter is a bounded set by Lemma 3.2 and hence $\Omega(x, \rho)$ is bounded.

Proposition 3.4 For each $\rho>1, x \mid A \backslash A_{\rho}$ is a finite total curvature minimal surface with only finitely many branch points; in particular, $x$ has an immersed end.

Proof: Branch points being isolated points, we may choose a radius $\sigma>\rho>1, \sigma$ arbitrarily large and $\rho$ arbitrarily close to 1 such that $x$ and $x_{R_{k}}$ are immersed along $\partial D_{\rho} \cup \partial D_{\sigma}$ for $k \geq k_{0}$. Denoting by $O(x, u)$ the order of a branch point $u$ of a minimal surface $x$ and setting $O(x, u)=0$ for regular points $u$, we obtain from the Gauss-Bonnet formula for branched minimal surfaces [D-H-K-W, Ch. 7.11]

$$
2 \pi \sum_{u \in A_{\sigma} \backslash A_{\rho}} O(x, u)-\iint_{A_{\sigma} \backslash A_{\rho}} K d \omega=\int_{\partial\left(A_{\sigma} \backslash A_{\rho}\right)} \kappa_{g} d s
$$

where $K$ and $d \omega$ denote the Gauss curvature and the area element of $x$ and $\kappa_{g}$ and $d s$ the geodesic curvature and the arclength element of the boundary curves $x\left(\partial\left(A_{\sigma} \backslash A_{\rho}\right)\right)$. By the smooth convergence of $x_{R_{k}}$ to $x$ on $\AA$ and again applying the Gauss-Bonnet formula on $x_{R_{k}}$ we obtain 


$$
\begin{aligned}
2 \pi \sum_{u \in A_{\sigma} \backslash A_{\rho}} O(x, u)-\iint_{A_{\sigma} \backslash A_{\rho}} K d \omega & =\lim _{k \rightarrow \infty} \int_{\partial\left(A_{\sigma} \backslash A_{\rho}\right)} \kappa_{g}^{k} d s^{k} \\
& =\lim _{k \rightarrow \infty}\left(2 \pi \sum_{u \in A_{\sigma} \backslash A_{\rho}} O\left(x^{k}, u\right)-\iint_{A_{\sigma} \backslash A_{\rho}} K^{k} d \omega^{k}\right) \\
& \leq \limsup _{k \rightarrow \infty}\left(2 \pi \sum_{u \in A_{r\left(R_{k}\right)} \backslash A_{\rho}} O\left(x^{k}, u\right)-\iint_{A_{r\left(R_{k}\right) \backslash A_{\rho}}} K^{k} d \omega^{k}\right) \\
& =\limsup _{k \rightarrow \infty} \int_{\partial\left(A_{\left.r\left(R_{k}\right) \backslash A_{\rho}\right)} \kappa_{g}^{k} d s^{k}\right.} \\
& \leq \limsup _{k \rightarrow \infty} \operatorname{curv}\left(\Gamma_{R_{k}}\right)-\int_{\partial D_{\rho}} \kappa_{g} d s,
\end{aligned}
$$

where $\operatorname{curv}\left(\Gamma_{R}\right)$ denotes the total absolute curvature of the curve $\Gamma_{R}$. It is however well known $[\mathrm{J}-\mathrm{M}]$ that $\frac{1}{R} \Gamma_{R}, \Gamma_{R}$ being the intersection of a finite total curvature end with a sphere of radius $R$, converges smoothly to a finite multiple of a great circle on the unit sphere. Hence $\operatorname{curv}\left(\Gamma_{R}\right)$ is bounded above independently of $R$ and the assertion of the lemma follows.

We may now state and prove our first main result,

Theorem 3.5 Let $\Gamma \subset B_{1}(0) \subset \mathbb{R}^{n}$ be a rectifiable Jordan curve and $T$ a twodimensional subspace of $\mathbb{R}^{n}$ which is not orthogonal to the affine subspace of minimal dimension containing $\Gamma$. Then there exists a complete, non-compact, area-minimizing annulus $M_{\infty}$ of finite total curvature spanning $\Gamma$ and possessing a flat end asymptotic to $T$ i. e. $M \backslash B_{R}(0)$ is a bounded graph over $T$ for a sufficiently large $R$.

Proof: We choose $M_{0}=T$ in the construction of section 2 above. Let $M_{\infty}$ be a limit surface of some sequence $\left(M_{R_{k}}\right)$. It follows from the convex hull property of minimal surfaces that each $M_{R}$ and hence $M_{\infty}$ is contained in the slab $S:=\left\{x \in \mathbb{R}^{n} \mid \operatorname{dist}(x, T) \leq 1\right\}$. Since by Proposition $3.4 M_{\infty}$ is known to be a properly immersed (possibly with branch points) finite total curvature surface it possesses a well defined asymptotic tangent space $T_{\infty}[H-O]$ and hence $\frac{1}{R} M_{\infty} \cap \partial B_{R}(0)$ converges smoothly to a multiple of the unit circle in $T_{\infty}[\mathrm{J}-\mathrm{M}]$. But $M_{\infty}$ being contained in the slab $S$, this unit circle must be contained in $T$ and, therefore, $T_{\infty}=T$. $M_{0}=T$ being a plane, the area estimates Lemma 2.5 and, hence, Lemma 3.1 hold with $m=1$. Now choose $R$ so big that all points of $M_{\infty} \backslash B_{R}(0)$ are regular for the orthogonal projection $P: \mathbb{R}^{n} \rightarrow T$. This is possible since $M_{\infty}$ has an immersed end by Proposition 3.4 and since $T$ is the asymptotic tangent plane of $M_{\infty}$. We may then conclude from the area estimate Lemma 3.1 (with $m=1$ ) that the degree of $P \mid M_{\infty} \backslash B_{R}(0)$ must be \pm 1 , i. e. $M_{\infty} \backslash B_{R}(0)$ has only one sheet over $T$.

\section{Flat ends of higher index}

In this section we use the concept of incompressible surfaces to find, for suitable Jordan curves, solutions to the exterior Plateau problem with a flat end of index $m$, i. e. the minimal surface remains at bounded distance to its asymptotic tangent plane at infinity and the orthogonal projection of the surface onto this plane has the topological degree $m \in \mathbb{N}$. As in the preceding 
sections, this solution is obtained as the limit of compact annuli where it is arranged that their projection map has the desired degree $m$. The main difficulty is of course to guarantee that in the limit the degree does not jump to a lower value. To accomplish this we make strong use of a barrier construction which enables us to apply the concept of incompressible surfaces. The barrier construction is made possible by our comparison principle for minimal submanifolds of arbitrary codimension $[\mathrm{J}-\mathrm{T}]$.

Our barriers are the boundaries of the domains

$$
D_{\lambda}=\left\{(z, w) \in \mathbb{R}^{2} \times \mathbb{R}^{n-2}|| w \mid \leq \varphi_{\lambda}(|z|)\right\}
$$

where $\varphi_{\lambda}$ is one of the catenaries

$$
\varphi_{\lambda}(t)=\lambda \ln \left(t / \lambda+\sqrt{t^{2} / \lambda^{2}-1}\right), t \geq \lambda>0
$$

It has already been shown in [J-T] that $\partial D_{\lambda}$ is "2-mean convex" with respect to the normal pointing into $D_{\lambda}$ and hence is a barrier for twodimensional minimal surfaces. We observe that $P\left(D_{\lambda}\right)=\left\{z \in \mathbb{R}^{2}|| z \mid \geq \lambda\right\}$, where $P: \mathbb{R}^{2} \times \mathbb{R}^{n-2} \rightarrow \mathbb{R}^{2}$ is the projection onto the first factor.

Let us consider a rectifiable Jordan curve $\Gamma \subset$ int $D_{\lambda}$ such that $P(\Gamma)$ has the winding number $m \in \mathbb{N}$ with respect to the origin in the $z$-plane and for any $R>1$ let $\Gamma_{R}$ denote the Jordan curve

$$
\Gamma_{R}(\zeta)=\left(\sqrt{R^{2}-1} \zeta^{m}, \zeta, 0, \ldots, 0\right), \zeta \in S^{1},
$$

where we split $\mathbb{R}^{n}$ as $\mathbb{R}^{n}=\mathbb{C} \times \mathbb{C} \times \mathbb{R}^{n-4}$.

We observe that $\Gamma_{R} \subset \partial B_{R}(0), \Gamma_{R} \subset$ int $D_{\lambda}$ for sufficiently big $R, P\left(\Gamma_{R}\right)$ has winding number $m$, and, furthermore

$$
\begin{aligned}
\operatorname{length}\left(\Gamma_{R}\right) & \leq m R, \\
\operatorname{curv}\left(\Gamma_{R}\right) & \leq 2 m \pi .
\end{aligned}
$$

It follows that, whenever $f: A_{r} \rightarrow D_{\lambda}$ is any continuous map such that $f \mid \partial A_{r}$ parametrizes $\Gamma-\Gamma_{R}$, then the induced map on fundamental groups $f_{*}: \pi_{1}\left(A_{r}\right) \rightarrow \pi_{1}\left(D_{\lambda}\right)$ is conjugate to the map $\mathbb{Z} \ni k \Rightarrow m k \in \mathbb{Z}$ and hence is injective. It follows from standard theory $[\mathrm{T}-\mathrm{T}]$ that there exists a branched minimal annulus $M^{R} \subset D_{\lambda}$ spanning $\Gamma-\Gamma_{R}$, parametrized by a conformal, branched, harmonic map $X^{R}: A_{r(R)} \rightarrow D_{\lambda}$ in the above conjugacy class and which minimizes area among all such maps. One easily constructs admissible comparison maps of area not larger $m R^{2} \pi+m R \pi+a_{0}$ with a fixed constant $a_{0}$, independent of $R$ and hence

$$
\operatorname{area}\left(M^{R}\right) \leq m R^{2} \pi+2 m R \pi+a_{0}
$$

The condition of cohesion (cf. Corollary (2.4)), is trivially satisfied for $M^{R}$ with $\varepsilon=2 m \pi \lambda$. From the above facts we may conclude that the theory of the preceding sections becomes applicable and that, for a suitable sequence $R_{k} \rightarrow \infty, X^{R_{k}}$ converges locally uniformly to a 
proper, branched, finite total curvature minimal surface $X: A \rightarrow D_{\lambda}$ spanning $\Gamma$. It follows immediately that the induced map $X_{*}$ on fundamental groups is conjugate to $k \mapsto m k$, and, since $P: D_{\lambda} \rightarrow D_{\lambda}$ is homotopic to the identity of $D_{\lambda}$, and $(P X)_{*}$ is conjugate to $k \mapsto m k$ as well. This proves that $P X$ has degree $m$ on the unbounded component of $P(\Gamma)$. It follows from the convex hull property of minimal surfaces that all of the $X^{R}$ and hence $X$ remain at bounded distance from the $z$-plane. Hence we proved

Theorem 4.1 Let $\Gamma \subset D_{\lambda}$ be a rectifiable Jordan curve such that $P \Gamma$ has the winding number $m$ around the origin, where $P: \mathbb{R}^{2} \times \mathbb{R}^{n-2} \rightarrow \mathbb{R}^{2}$ is the orthogonal projection. Then $\Gamma$ spans a complete finite total curvature minimal annulus $M \subset D_{\lambda}$ with a flat end and the z-plane as its asymptotic tangent space. Moreover, $P \mid M$ has the topological degree $m$.

\section{Surfaces with a polynomial end}

In this section we choose as our model surface $M_{0}$ the complex curve

$$
w=z^{m}
$$

where $(z, w) \in \mathbb{C} \times \mathbb{C} \cong \mathbb{R}^{4}$ and $m \in \mathbb{N}$.

For some sufficiently large $R>0$ we shall construct a neighborhood $B$ of $M_{0} \cap\{|w|>R\}$ which lies within bounded distance of $M_{0}$ and which has 2-mean convex boundary $\partial B$. Since $B$ has the homotopy type of the circle, the methods of the previous paragraphs yield the existence of complete minimal annuli within $B$ spanning a given homotopically nontrivial Jordan curve in $B$.

The asymptotical tangent plane at infinity of $M_{0}$ being the $w$-plane, we prefer, simultaneously interchanging $z$ and $w$, to write (5.1) in the equivalent form

$$
w=z^{1 / m},
$$

thus representing the surface as an $m$-sheeted graph (outside of the origin) over its asymptotical tangent plane. Locally we may write (5.2) as a single valued graph

$$
z=x_{1}+i x_{2}, w=u_{1}+i u_{2}
$$

where $u_{1}$ and $u_{2}$ are functions of $\left(x_{1}, x_{2}\right)$ which are solutions of the Cauchy- Riemann equations

$$
D_{1} u_{1}=D_{2} u_{2}, D_{2} u_{1}=-D_{1} u_{2}, D_{j}=\frac{\partial}{\partial x_{j}}
$$

An orthogonal basis $\left(e_{1}, e_{2}\right)$ of the tangent bundle and an orthonormal basis $\left(N_{1}, N_{2}\right)$ of the normal bundle of $M_{0}$ are therefore given by

$$
e_{1}=\left(1,0, D_{1} u_{1}, D_{1} u_{2}\right), e_{2}=\left(0,1, D_{2} u_{1}, D_{2} u_{2}\right)
$$


and

$$
N_{1}=\lambda^{-1}\left(-D_{1} u_{1}, D_{2} u_{2}, 1,0\right), N_{2}=\lambda^{-1}\left(D_{2} u_{1},-D_{2} u_{2}, 0,1\right)
$$

where $\lambda=\sqrt{1+\left|D u_{1}\right|^{2}}=\sqrt{1+\left|D u_{2}\right|^{2}}$. Since

$$
\left|D u_{j}\right|=\frac{1}{m} \rho^{1 / m-1},\left|D^{2} u_{j}\right| \leq c \rho^{1 / m-2}
$$

with $\rho=|z|=\sqrt{x_{1}^{2}+x_{2}^{2}}$ we obtain

$$
\begin{aligned}
& \left|D_{j} e_{k}\right| \leq c \rho^{1 / m-2},\left|\left\langle D_{j} e_{k}, e_{\ell}\right\rangle\right| \leq c \rho^{2 / m-3} \\
& \left|D_{j} N_{k}\right| \leq c \rho^{1 / m-2},\left|D_{i j} N_{k}\right| \leq c \rho^{1 / m-3}
\end{aligned}
$$

the constant $c$ depending only on $M_{0}$.

As a first step we seek the desired neighborhood $B$ of $M_{0}$ in the form

$$
B=\left\{(z, w) \in \mathbb{C}^{2} \mid \quad \operatorname{dist}\left((z, w), M_{0}\right) \leq f(|z|)\right\}
$$

where $f$ is still to be determined. In order to calculate the second fundamental form of $\partial B$ we choose a local representation of $M_{0}$ of the form (5.3) which we denote by $X$. A local parametrization $Y$ of $\partial B$ is then obtained as

$$
Y\left(x_{1}, x_{2}, N\right)=X\left(x_{1}, x_{2}\right)+f(\rho) N
$$

where $N$ varies in the normal unit sphere of $M_{0}$ at $\left(x_{1}, x_{2}\right)$. The computations to follow are facilitated by means of

Lemma 5.1 Given any point $p \in M_{0}$ we may locally modify the basis $\left(N_{1}, N_{2}\right)$ in such a way that the relations

$$
\left\langle D_{k} N_{i}, N_{j}\right\rangle=0
$$

hold at $p$. The estimates (5.4) remain valid.

Proof: We define the modified basis by $\tilde{N}_{1}=\cos \varphi N_{1}+\sin \varphi N_{2}, \tilde{N}_{2}=-\sin \varphi N_{1}+\cos \varphi N_{2}$ with a function $\varphi=\varphi\left(x_{1}, x_{2}\right)$ yet to be determined. The new basis being again orthonormal, it suffices to consider the case $i=1, j=2$ in (5.7). One finds

$$
\left\langle D_{k} \tilde{N}_{1}, \tilde{N}_{2}\right\rangle=D_{k} \varphi+\left\langle D_{k} N_{1}, N_{2}\right\rangle
$$

and we may therefore choose $\varphi$ as a first order polynomial in $\left(x_{1}, x_{2}\right)$ such that $\varphi(p)=0$ and $D_{k} \varphi(p)=-\left\langle D_{1} N_{1}(p), N_{2}(p)\right\rangle$.

We start by calculating a local basis $\left(T_{1}, T_{2}, T_{3}\right)$ for the tangent bundle of $\partial B$, substituting $N=\cos \theta N_{1}+\sin \theta N_{2}$ :

$$
\begin{aligned}
& T_{j}=D_{j} Y=e_{j}+f^{\prime}(\delta) D_{j} \rho\left(\cos \theta N_{1}+\sin \rho N_{2}\right)+f\left(\cos \theta D_{j} N_{1}+\sin \theta D_{j} N_{2}\right), \quad(j=1,2), \\
& T_{3}=D_{\theta} Y=f\left(-\sin \theta N_{1}+\cos \theta N_{2}\right)
\end{aligned}
$$


Shifting the angular variable $\theta$ we may assume that $\theta=0$ at the point under consideration. The metric tensor $\left(g_{i j}\right)$ then becomes

$$
\begin{array}{rrr}
g_{i j}=\lambda^{2} \delta_{i j}+2 f\left\langle D_{i} N_{1}, e_{j}\right\rangle+f^{\prime 2} D_{i} \rho D_{j} \rho+f^{2}\left\langle D_{i} N_{1}, D_{j} N_{1}\right\rangle & (i, j=1,2), \\
g_{i 3}=0 & (i=1,2) \\
g_{33}=f^{2} . &
\end{array}
$$

Introducing the normalizations

$$
0 \leq f \leq 1,\left|f^{\prime}\right| \leq 1, \quad \rho \geq 1
$$

we obtain from (5.5) and (5.6)

$$
g_{i j}=\lambda^{2} \delta_{i j}+O\left(\rho^{1 / m-2} f+f^{\prime 2}\right),
$$

Here and in what follows the growth rate of the $O$-term depends only on $M_{0}$, always taking (5.8) into account. For the inverse matrix we then get

$$
\begin{aligned}
g^{i j} & =\lambda^{-2} \delta_{i j}+O\left(\rho^{1 / m-2} f+f^{\prime 2}\right) \\
g^{i 3} & =0 \\
g^{33} & =f^{-2} .
\end{aligned}
$$

A normal vector $\nu$ for $\partial B$ may be written in the form

$$
\nu=\alpha_{1} e_{1}+\alpha_{2} e_{2}+\cos \theta N_{1}+\sin \theta N_{2}
$$

the coefficients $\alpha_{j}$ being determined from the relations

$$
0=\left\langle\nu, T_{j}\right\rangle=\lambda^{2} \alpha_{j}+\sum_{i=1,2} f \alpha_{i}\left(\left\langle D_{j} N_{1}, e_{i}\right\rangle \cos \theta+\left\langle D_{j} N_{2}, e_{i}\right\rangle \sin \theta\right)+f^{\prime} D_{j} \rho \quad(j=1,2)
$$

After an orthogonal coordinate transformation we may assume that

$$
\left\langle D_{i} N_{i}, e_{j}\right\rangle=\kappa_{i}\left\langle e_{i}, e_{j}\right\rangle=\kappa_{i} \lambda^{2} \delta_{i j},
$$

the $\kappa_{i}$ being the principal curvatures of $M_{0}$ in direction $N_{1}$. Our normalization $\theta=0$ and (5.10) lead to

$$
\alpha_{j}=-\lambda^{-2}\left(1+f \kappa_{j}\right)^{-1} f^{\prime} D_{j} \rho
$$


assuming from now on $\rho \geq \rho_{0}$ to ensure $1+f \kappa_{j} \geq \frac{1}{2}$. Continuing towards the calculation of the second fundamental form we need

$$
\begin{aligned}
D_{j} T_{i}= & D_{j} e_{i}+\left(f^{\prime \prime}(\rho) D_{i} \rho D_{j} \rho+f^{\prime}(\rho) D_{i j} \rho\right)\left(\cos \theta N_{1}+\sin \theta N_{2}\right)+f^{\prime}(\rho) D_{i} \rho\left(\cos \theta D_{j} N_{1}+\right. \\
& \left.\sin \theta D_{j} N_{2}\right)+f^{\prime}(\rho) D_{j} \rho\left(\cos \theta D_{i} N_{1}+\sin \theta D_{i} N_{2}\right)+f(\rho)\left(\cos \theta D_{i j} N_{1}+\right. \\
& \left.\sin \theta D_{i j} N_{2}\right), \\
D_{3} T_{i}= & D_{\theta} T_{i}=f^{\prime}(\rho) D_{i} \rho\left(-\sin \theta N_{1}+\cos \theta N_{2}\right)+f(\rho)\left(-\sin \theta D_{i} N_{1}+\cos \theta D_{i} N_{2}\right), \quad(i=1,2), \\
D_{3} T_{3}= & D_{\theta} T_{3}=f\left(-\cos \theta N_{1}-\sin \theta N_{2}\right) .
\end{aligned}
$$

Again using (5.7), (5.10) and the normalization $\theta=0$ we calculate $b_{i j}:=-\left\langle\nu, D_{j} T_{i}\right\rangle$ as follows:

$$
\begin{array}{rlr}
b_{i j}= & -\sum_{k=1,2} \alpha_{k}\left\langle D_{j} e_{i}, e_{k}\right\rangle-f^{\prime}(\rho) D_{i} \rho \alpha_{j} \kappa_{j} \lambda^{2}-f^{\prime}(\rho) D_{j} \rho \alpha_{i} \kappa_{i} \lambda^{2}+\lambda^{2} \kappa_{i} \delta_{i j} & \\
& -f^{\prime \prime}(\rho) D_{i} \rho D_{j} \rho-f^{\prime}(\rho) D_{i j} \rho-f\left\langle\sum \alpha_{k} e_{k}+N_{1}, D_{i j} N_{1}\right\rangle, & (i, j=1,2), \\
b_{i 3}= & -\sum_{k=1,2} \alpha_{k} f\left\langle D_{i} N_{2}, e_{k}\right\rangle & (i=1,2), \\
b_{33}= & f . &
\end{array}
$$

By means of (5.4), and (5.10) we obtain the estimates

$$
\begin{aligned}
b_{i j}= & \lambda^{2} \kappa_{i} \delta i j-\left(f^{\prime \prime}(\rho) D_{i} \rho D_{j} \rho+f^{\prime} D_{i j} \rho\right) \\
& +O\left(\rho^{2 / m-3} f^{\prime}+\rho^{1 / m-2} f^{\prime 2}+\rho^{2 / m-4}\right), \\
b_{i 3}= & O\left(\rho^{1 / m-2} f^{\prime} f\right) \\
b_{33}= & f .
\end{aligned}
$$

Let us now consider the submatrices

$$
G=\left(g^{i j}\right)_{i j=1,2}, B=\left(b_{i j}\right)_{i, j=1,2} .
$$

(5.19) and (5.11) yield the following representation for the second fundamental tensor $A$ in direction $\nu$ :

$$
A=\left(g^{i j}\right)\left(b_{i j}\right)=A_{0}+O\left(\rho^{1 / m-2}\left(f f^{\prime}+f^{\prime} / f\right)\right)
$$


with

$$
A_{0}=\left(\begin{array}{ccc}
G & B & 0 \\
& & 0 \\
0 & 0 & 1 / f
\end{array}\right) .
$$

Since $G=\lambda^{-2} I+O\left(f^{\prime 2}+\rho^{1 / m-2}\right)$ and $B=O\left(\rho^{1 / m-2}+\left|f^{\prime \prime}\right|+\rho^{-1}\left|f^{\prime}\right|\right)$ we conclude that

$$
G B=\lambda^{-2} B+O\left(\left(f^{\prime 2}+\rho^{1 / m-2}\right)\left(\rho^{1 / m-2}+\left|f^{\prime \prime}\right|+\left|f^{\prime}\right| / \rho\right)\right) .
$$

Let us now choose

$$
f(\rho)=1-\rho^{-\beta}
$$

with $\beta \in] 0,2\left[\right.$. Using the minimality of $M_{0}$ we obtain

$$
\text { trace } B=-\left(f^{\prime \prime}(\rho)+\frac{1}{\rho} f^{\prime}(\rho)\right)+O\left(\rho^{2 / m-3} f^{\prime}+\rho^{1 / m-2} f^{\prime 2}+\rho^{2 / m-4}\right)=\rho^{-2-\beta}+O\left(\rho^{2 / m-4}\right) \text {. }
$$

Together with (5.15) this yields the estimate for the eigenvalues $\lambda_{i}(G B)$

$$
\begin{aligned}
\lambda_{1}(G B)+\lambda_{2}(G B) & =\operatorname{trace}(G B) \\
& =\lambda^{-2} \rho^{-2-\beta}+O\left(\rho^{2 / m-4}\right) .
\end{aligned}
$$

The eigenvalues $\lambda_{i}\left(A_{0}\right)$ being

$$
\lambda_{1}\left(A_{0}\right)=\lambda_{1}(G B), \lambda_{2}\left(A_{0}\right)=\lambda_{2}(G B), \lambda_{3}\left(A_{0}\right)=1 / f,
$$

where $\lambda_{i}\left(A_{0}\right)=O(\|B\|)=O\left(\rho^{1 / m-2}\right)$ for $i=1,2$, we see immediately that

$$
\begin{aligned}
\lambda_{1}\left(A_{0}\right)+\lambda_{2}\left(A_{0}\right) & \geq \frac{1}{2} \lambda^{-2} \rho^{-2-\beta} \\
\lambda_{i}\left(A_{0}\right)+\lambda_{3}\left(A_{0}\right) & \geq \frac{1}{2} f(\rho)^{-1} .
\end{aligned}
$$

provided that we choose $\beta \in] 0,2-2 / m\left[\right.$ and $\rho \geq \rho_{0}, \rho_{0}$ depending on $M_{0}$ and $\beta$. It remains to estimate the difference between the eigenvalues $\lambda_{i}(A)$ and $\lambda_{i}\left(A_{0}\right)$. We have $f^{\prime}(\rho) / f(\rho) \leq$ $2 \beta \rho^{-1-\beta}$ for $\rho \geq 2^{1 / \beta}$ and hence we conclude from (5.12) that

$$
\lambda_{i}(A)-\lambda_{i}\left(A_{0}\right)=O\left(\rho^{1 / m-3-\beta}\right) .
$$


Comparing (5.15) and (5.16) we see that the sum of any two eigenvalues of $A$ is positive for $\rho \geq \rho_{0}$, proving the 2-mean convexity of $\partial B$ outside of the cylinder $x_{1}^{2}+x_{2}^{2} \leq \rho_{0}^{2}$. Let us remark that all our estimates being direct, the bound $\rho_{0}$ can be computed explicitly.

Since we can show the 2-mean convexity of the boundary of the set $B$ defined in (5.5) only for $|z| \geq \rho_{0}$ for some sufficiently large $\rho_{0}$ it becomes necessary to modify the definition of $B$ in order to obtain a set with everywhere 2 -mean convex boundary. Consequently we define

$$
B=B_{\rho} \cup T_{\rho}
$$

with

$$
B_{\rho}=\left\{(z, w)\left|\operatorname{dist}\left((z, w), M_{0}\right) \leq f(|z|),\right| z \mid \geq \rho\right\}
$$

and $T_{\rho}$ essentially being a tubular neighborhood of the curve $C_{\rho}=M_{0} \cap\{|z|=\rho\}$ Introducing polar coordinates $(\rho, \varphi)$ in the $z$-plane and a further angular variable $\psi$ we parameterize $\partial T_{\rho}$ by the map

$$
Z_{\rho}(s, N, \psi)=X(\rho, \varphi)+\delta g(\psi) N+h(s) \sin \psi D_{\rho} X(\rho, \varphi)
$$

where $s=\rho \varphi, 0 \leq \varphi \leq 2 m \pi, \pi / 2 \leq \psi \leq \pi / 2, N$ varies in the normal unit sphere bundle of $M_{0}$ restricted to $C_{\rho}, \delta>0$, and $g$ as well as $h$ are yet to be determined with $g(\psi)=\cos \psi$ near $\psi= \pm \pi / 2$. Let us remark that $\psi= \pm \pi / 2$ does not introduce any boundary in (5.19) since the curve $Z_{\rho}(s, N, \cdot)$ closes up withe $Z_{\rho}(s,-N, \cdot)$. The plan is to glue half of $\partial T_{\rho}$, i. e. the portion of $\partial T_{\rho}$ corresponding to $\psi \in[-\pi / 2,0]$ to $5.14 \partial B_{\rho}$ along the surface $Z_{\rho}(\cdot, \cdot, 0)$ for some large $\rho=\rho_{0}, B_{\rho}$ being defined in (5.18). The gluing condition of order zero is

$$
Z_{\rho}(\rho \varphi, N, 0)=Y(\rho, \varphi, N)
$$

which is equivalent to

$$
\delta g(0)=f(\rho)
$$

It will be sufficient to make the gluing of class $C^{1}$, simultaneously keeping the pieces $\partial B_{\rho}$ and $\partial T_{\rho}$ strictly 2-mean convex, because then $\partial B_{\rho} \cup \partial T_{\rho}$ may be further smoothened in a standard way.

Let us start by computing a local basis $\left(V_{1}, V_{2}, V_{3}\right)$ of the tangent bundle of $\partial T_{\rho}$ :

$$
\begin{aligned}
V_{1} & :=D_{s} Z_{\rho}=\frac{1}{\rho} D_{\varphi} Z_{\rho}=\frac{1}{\rho}\left\{D_{\varphi} X+\delta g(\psi)\left(\cos \theta D_{\varphi} N_{1}+\sin \theta D_{\varphi} N_{2}\right)\right. \\
& \left.+\rho h^{\prime}(s) \sin \psi D_{\rho} X+h \sin \psi D_{\rho \varphi} X\right\}
\end{aligned}
$$




$$
\begin{aligned}
& V_{2}:=D_{\theta} Z_{\rho}=\delta g(\psi)\left(-\sin \theta N_{1}+\cos \theta N_{2}\right) \\
& V_{3}:=D_{\psi} Z_{\rho}=\delta g^{\prime}(\psi)\left(\cos \theta N_{1}+\sin \theta N_{2}\right)+h \cos \psi D_{\rho} X
\end{aligned}
$$

Comparing with (5.6) at $\theta=0, \psi=0$ and, besides (5.20) also requiring that

$$
\delta g^{\prime}(0)=f^{\prime}(\rho)
$$

we see that

$$
\begin{aligned}
& D_{\varphi} Y=\rho V_{1}, D_{\theta} Y=V_{2}, \\
& D_{\rho} Y=D_{\rho} X+f^{\prime} N_{1}+f D_{\rho} N_{1}, \\
& V_{3}=h D_{\rho} X+f^{\prime} N_{1} .
\end{aligned}
$$

Hence, in order that the tangent spaces of $\partial B_{\rho}$ and $\partial T_{\rho}$ along the glueing surface coincide we must adjust $h$ in such a way that $D_{\rho} Y$ becomes a linear combination of $V_{1}, V_{2}$, and $V_{3}$. According to Lemma 5.1 we may write

$$
D_{\rho} N_{1}=\alpha_{1} D_{\rho} X+\alpha_{2} D_{\varphi} X, D_{\varphi} N_{1}=\beta_{1} D_{\rho} X+\beta_{2} D_{\varphi} X
$$

with

$$
\begin{aligned}
& \alpha_{1}=\left|D_{\rho} X\right|^{-2}\left\langle D_{\rho} N_{1}, D_{\rho} X\right\rangle, \alpha_{2}=\left|D_{\varphi} X\right|^{-2}\left\langle D_{\rho} N_{1}, D_{\varphi} X\right\rangle \\
& \beta_{1}=\left|D_{\rho} X\right|^{-2}\left\langle D_{\varphi} N_{1}, D_{\rho} X\right\rangle, \beta_{2}=\left|D_{\varphi} X\right|^{-2}\left\langle D_{\varphi} N_{1}, D_{\varphi} X\right\rangle .
\end{aligned}
$$

From this we obtain

$$
\begin{aligned}
& D_{\varphi} X=\left(1+\beta_{2} f\right)^{-1} V_{1}-f \beta_{1}\left(1+\beta_{2} f\right)^{-1} D_{\rho} X, \\
& D_{\rho} N_{1}=\left(\alpha_{1}-f \beta_{1}\left(1+\beta_{2} f\right)^{-1}\right) D_{\rho} X+\alpha_{2}\left(1+\beta_{2} f\right)^{-1} V_{1},
\end{aligned}
$$

and hence

$$
D_{\rho} Y=\left(1+f\left(\alpha_{1}-f \beta_{1}\left(1+\beta_{2} f\right)^{-1}\right) D_{\rho} X+f^{\prime} N_{1}+f \alpha_{2}\left(1+\beta_{2} f\right)^{-1} V_{1},\right.
$$

from which we see that we must choose

$$
h=1+f(\rho)\left(\alpha_{1}-f(\rho) \beta_{1}\left(1+\beta_{2} f(\rho)\right)^{-1}\right),
$$

$\alpha_{j}, \beta_{j}$ being functions of the angle $\varphi$.

Using 


$$
\begin{aligned}
& \left|D_{\rho} X\right|^{2}=1+\frac{1}{m^{2}} \rho^{2 / m-2},\left|D_{\varphi} X\right|^{2}=\rho^{2}\left|D_{\rho} X\right|^{2} \\
& \left|D_{\rho \rho} X\right|^{2}=\frac{(m-1)^{2}}{m^{4}} \rho^{2 / m-4},\left|D_{\varphi \varphi} X\right|^{2}=\rho^{2}\left(1+\frac{1}{m^{4}} \rho^{2 / m-2}\right) \\
& \left|D_{\rho \varphi} X\right|^{2}=1+\frac{1}{m^{4}} \rho^{2 / m-2} \\
& \left|D_{\rho} N_{j}\right|=O\left(\rho^{1 / m-2}\right),\left|D_{\varphi} N_{j}\right|=O\left(\rho^{1 / m-1}\right)
\end{aligned}
$$

one verifies that $\alpha_{j}$ and $\beta_{j}$ as well as their derivatives with respect to $\varphi$ are at most of the order $O\left(\rho^{1 / m-1}\right)$ and hence, keeping $s=\rho \varphi$ in mind,

$$
\begin{aligned}
& h=1+O\left(\rho^{1 / m-1}\right), h^{\prime}=O\left(\rho^{1 / m-2}\right) \\
& h^{\prime \prime}=O\left(\rho^{1 / m-3}\right) .
\end{aligned}
$$

Let us now define the function $g$. We choose $\eta \in C^{\infty}(\mathbb{R})$ with $|\eta| \leq 1, \eta(\psi)=0$ for $\psi \leq$ $-\pi / 4, \eta(0)=0, \eta^{\prime}(0)=1$ and, remembering that $f(\rho)=1-\rho^{-\beta}$, we define

$$
g(\psi)=\cos \psi+\beta \rho^{-1-\beta}\left(1-\rho^{-\beta}\right)^{-1} \eta(\psi) .
$$

Setting $\delta:=1-\rho^{-\beta}$ we see that (5.20) and (5.22) are fulfilled. Moreover,

$$
D_{\psi}^{j}(g(\psi)-\cos \psi)=O\left(\rho^{-1-\beta}\right) \text { for } j=0,1,2 .
$$

We would like to argue that, for large $\rho,(5.19)$ is a $C^{2}$-small perturbation of the standard round tubular neighborhood of radius 1 of the curve $X(\rho, \cdot)$ where $\delta=1$ and $h=\left|D_{\rho} X\right|^{-1}=$ $1 / \sqrt{1+\frac{1}{m^{2}} \rho^{2 / m-2}}$, implying that (5.19) would be 2-mean convex for sufficiently large $\rho$, since this is certainly the case for the standard tube if $\rho$ is sufficiently large. The $C^{2}$ closeness is obvious for the mapping $Z_{\rho}$ on account of (5.24) and (5.25). In order to conclude the closeness of the second fundamental forms one must make sure that the first fundamental forms remain uniformly nonsingular. This does not seem to be case near $\psi=-\pi / 2$ where $V_{2}$ in (5.23) becomes zero. But this this is simply a deficiency of our coordinate system which disappears if we introduce new coordinates in the range $\left[-\frac{\pi}{2}, \frac{\pi}{4}\right]$ according to

$$
x_{1}=\cos \psi \cos \theta, x_{2}=\cos \psi \sin \theta
$$

transforming (5.19) into

$$
Z_{\rho}=X(\rho, \varphi)+\delta\left(x_{1} N_{1}+x_{2} N_{2}\right)-h(s) \sqrt{1-\left(x_{1}^{2}+x_{2}^{2}\right)} D_{\rho} X(\rho, \varphi) .
$$

It is easily seen that the metric tensor of $Z_{\rho}$ in the coordinates $\left(s=\rho \varphi, x_{1}, x_{2}\right)$ is uniformly nonsingular for large $\rho$ in the range $x_{1}^{2}+x_{2}^{2} \leq 1 / 2$. Hence we proved 
Theorem 5.2 There is an explicitly computable number $\rho_{0}>0$ so that the surface

$$
M_{\rho_{0}}:=\left\{(z, w) \in \mathbb{C}^{2}\left|w=z^{m},\right| w \mid \geq \rho_{0}\right\}
$$

admits a neighborhood $B$ with 2-mean convex boundary. $B$ retracts onto $M_{\rho_{0}}$ and the points of $B$ are within distance 1 from $M_{\rho_{0}}$, the distance between $\partial B$ and $M_{\rho_{0}}$ approaching 1 at infinity.

We remark that the distance 1 condition is just a normalization; the number 1 can be replaced by any positiv number, however with the effect that $\rho_{0}$ has to be adjusted. On the basis of Theorem 5.2 we finally obtain the following existence theorem along the same lines as in section 4.

Theorem 5.3 Let $M_{\rho_{0}}$ and $B$ as in Theorem 5.2. Then, given any rectifiable Jordan curve $\Gamma$ in $B$ which is freely homotopic in $B$ to $\partial M_{\rho_{0}}$, there exists a complete annulus type minimal surface $M$ in $B$ with boundary $\Gamma$ and finite total curvature end. In particular, $M$ stays within distance 1 from $M_{\rho_{0}}$ and hence $M$ behaves like $w=z^{m}$ at infinity. 


\section{Bibliography}

[C] Courant, R., Dirichlet's principle, conformal mapping, and minimal surfaces. Reprint, Springer 1977, New York, Heidelberg, Berlin

[D] Douglas, J., The problem of plateau for two contours, Journal of Math. and Physics 10, 310-359

[D-H-K-W] Dierkes, U., Hildebrandt, S., Küster, A., Wohlrab, O., Minimal Surfaces I, II. Springer 1992, Berlin, Heidelberg, New York

[E-W-W] Ekholm, T., White, B., Wienholtz, D., Embeddedness of minimal

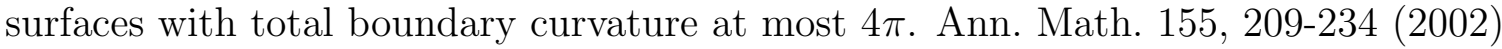

[H-O] Hoffmann, D. A., Osserman, R., The geometry of the generalized Gauss map, Mem. Am. Math. Soc. vol. 28, no. 236, 1980

[J- M] Jorge, P., L., Meeks, W. H. , The topology of complete minimal surfaces of finite total Gaussian curvature, Topology 22, 203-221 (1983)

[J-T] Jorge, P. L. Tomi, F., The barrier principle for minimal submanifolds of arbitrary codimension, preprint, 2002

[M] Morgan, F., On the singular structure of two-dimensional area minimizing surfaces in $\mathbb{R}^{n}$, Math. Ann. 261, 101-110 (1982)

[T-T] Tomi, F., Tromba, A. J., Existence theorems for minimal surfaces of non-zero genus spanning a contour, Mem. Am. Math. Soc. vol. 71, no. 382, 1988

[T-Ye] Tomi, F., Ye, R., The exterior Plateau problem, Math. Z. 205, 233-245 (1990) 